\section{Ahmed Glaucoma Valve Implantation in the Surgical Treatment of Traumatic Glaucoma - Case Report Malgorzata Mrugacz* and Anna Bryl}

Department of Pediatric Ophthalmology, Department of Eye Rehabilitation, Medical University of Bialystok, Bialystok, Poland

\begin{abstract}
To report the safety and efficacy of Ahmed Glaucoma Valve implantation for the management of traumatic glaucoma. An 11 -year-old girl with posttraumatic megalocornea and buphthalmos underwent an Ahmed valve implantation in her left eye. The patient was operated before because of corneal injury and then she underwent iridectomy and had performed cyclocryotherapy. The intraocular pressure was $24-60 \mathrm{mmHg}$ with maximal medical therapy by this time. Mean postoperative IOP was $35 \mathrm{mmHg}$ during the first week. After a period of relatively low IOP during the next 7 months (5-10 $\mathrm{mmHg})$, pressure values reached a plateau of around 14-16 $\mathrm{mmHg}$. We observed hypotony, tube exposure and fibrotic reaction around the valve postoperatively. The Ahmed Glaucoma valve may be safe and effective treatment for traumatic glaucoma in children, despite its postoperative complications. This procedure may be used to treat complicated glaucoma in eyes with previous failed filtering procedures.
\end{abstract}

Keywords: Ahmed Valve; Children; Glaucoma; Trauma

Glaucoma drainage implants, including the Ahmed Glaucoma Valve (New World Medical, Rancho Cucamonga, California, USA) have been used to treat complicated glaucomas such as aphakic or pseudophakic glaucoma, neovascular glaucoma, refractory glaucoma, no response to other glaucoma surgery, glaucoma associated with uveitis, glaucoma in Sturge -Weber syndrome, glaucoma following penetrating keratoplasty and traumatic and refractory glaucoma. In children, drainage implants have been used as an alternative to filtration surgery when angle surgery, such as goniotomy and trabeculotomy, has failed [1-6]. The Ahmed Glaucoma Valve Implant consists of a silicone tube connected to a silicone sheet valve held in a polypropylene body. The valve is designed to open at a pressure of 8 $\mathrm{mmHg}$, decreasing the risk of postoperative hypotony.

*Corresponding author: Małgorzata Mrugacz, Department of Pediatric Ophthalmology, Department of Eye Rehabilitation, Medical University of Bialystok, 17 Washington Str, 15-274 Białystok, Poland, Tel: +48 85 7450862; Fax: +48 85750858; E-mail: malgorzata.mrugacz@umb.edu.pl

Citation: Malgorzata M, Anna B (2015) Ahmed Glaucoma Valve Implantation in the Surgical Treatment of Traumatic Glaucoma - Case Report. J Ophthalmic Clin Res 2: 004.

Received: August 27, 2014; Accepted: January 08, 2014; Published: January 22, 2015
This case report describes the safety and efficacy of Ahmed Glaucoma Valve implantation for the management of traumatic glaucoma.

\section{Case Report}

An 11-year-old girl with posttraumatic magalocornea and buphthalmos underwent an Ahmed valve implantation in her left eye. Informed consent was obtained from the parents before this surgery. The patient was operated before because of corneal injury and then she underwent iridectomy and had performed cyclocryotherapy. The intraocular pressure was $24-60 \mathrm{mmHg}$ with maximal medical therapy by this time. The procedure of Ahmed Glaucoma Valve implantation used a standardized surgical technique [1,2,7].

The postoperative regimen included a topical antibiotic and a cycloplegic for 2 to 4 weeks, and topical steroids for approximately 2 months. Anti-glaucoma medication was added as required to improve IOP reduction for the first week. Patient was examined 1 day, 7 days, 14 days, 1 month, 2 months, 3 months, 6 months (Figure 1) and 12 months after surgery. Surgical success was defined as postoperative IOP level between 10 and $21 \mathrm{mmHg}$.

On ocular examination, visual acuity was RE: 20/20 and LE: no light perception. Mean postoperative IOP in left eye was $35 \mathrm{mmHg}$ during the first week and $16 \mathrm{mmHg}$ at 12 months. We observed hyphema on the first postoperative day and hypotony 3 weeks after surgery. Hypotonywas defined as an IOPless than $5 \mathrm{mmHg}$ or shallowing of the anterior chamber without evidence of aqueous misdirection. This resolved within 1 month. Tube exposure and fibrotic reaction around the valve occurred 3 months postoperatively. The tube was repositioned surgically and fibrotic reaction resolved within 3 weeks.

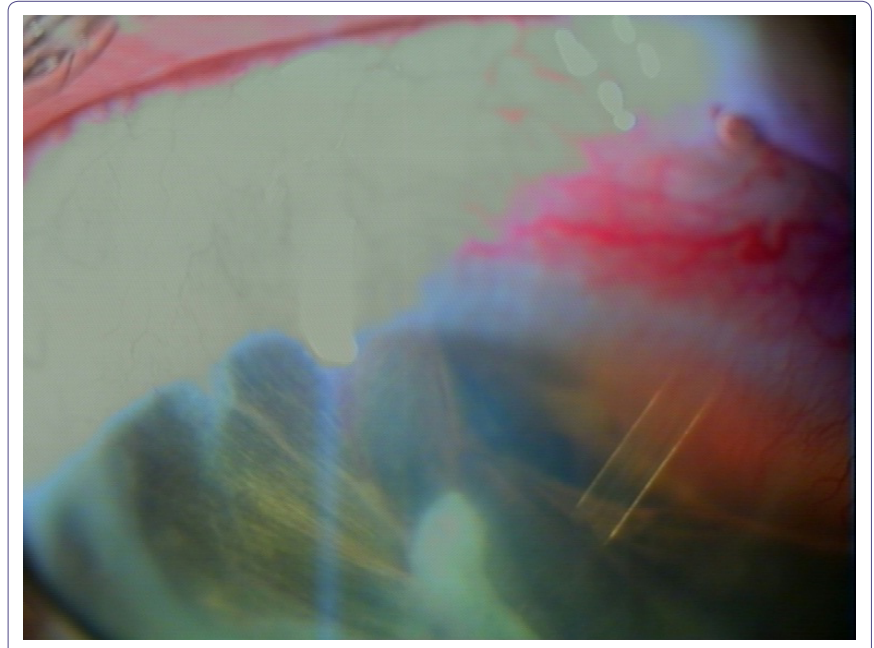

Figure 1: Ahmed Glaucoma Valve implant 6 months after surgery.

\section{Discussion}

Management of pediatric glaucoma, especially traumatic glaucoma that does not respond satisfactorily to medical treatment or filtering surgery is very difficult. The higher failure rate of trabeculectomy in 
children and long-term complications of intraoperative antimetabolites warrant consideration of alternative options [2]. Glaucoma drainage devices are used frequently to control intraocular pressure in complicated glaucomas. We have therefore explored the role of Ahmed implant insertion in our pediatric patient with traumatic glaucoma.

The reported success rate of drainage implants in children varies from $44 \%$ to $95 \%$. Coleman et al., [7] reported an overall success rate of $77 \%$ and $60.6 \%$, Englert et al., [8] reported a success rate of $90.6 \%$ and $58.3 \%$ at 12 and 24 months, respectively, and Djodeyre et al., [2] reported an overall success rate of $70 \%$ to $63 \%$ at 12 and 24 months, respectively in pediatric patients with Ahmed Glaucoma Valve implants. Recently, Morad et al., [1] reported a success rate of $71 \%$ to $46 \%$ after 36 and 48 months, respectively, and Ozdal et al., [4] reported a success rate of $94.4 \%$ at 1 year and $60 \%$ at 2 years. Hamush et al., [3] reported a 79\% and 30\% success rate in controlling IOP at 12 and 60 months, respectively, and Morad et al., [1] reported an overall success rate of $100 \%$ to $85 \%$ at 1 year and 5 years, respectively in the population of patients with Sturge-Weber glaucoma.

A period of transient elevation of intraocular pressure, known as hypertensive phase, has been described after glaucoma drainage implant surgery, appearing approximately 4 weeks after surgery and lasting at least 12 to 16 weeks [9]. In our patient the intraocular pressure was greater than $21 \mathrm{mmHg}$ during the first week after surgery. Coleman et al., [7] found a moderately higher intraocular pressure during postoperative months 4 to 6 with Ahmed glaucoma Valve implants in adults.

Postoperative hypotony which appears in our patient 3 weeks after surgery can be a major problem with all aqueous drainage implants affecting surgical outcome. The study by Francis et al., [10] have shown that the Ahmed glaucoma Valve, as opposed to the Optimed or Krupin implants, has the best flow-restricting function and closely regulates pressure within a desired range. Initial reports with the Ahmed valve in children reported this complication from $11 \%$ to $14 \%[7,8]$. In the larger series reported by Djodeyre et al., [2], the hypotony rate was $25 \%$, similar to the results of $23 \%$ according to the study by Morad et al., [1].

In our patient we found hyphema on the first postoperative day. According to the studies by Ayyala et al., [9], Wu et al., [5], and Montanez et al., [6] hyphema is the most common early postoperative complication in complicated glaucoma with the Ahmed glaucoma valve.

Several clinical studies have identified as late complication tube malposition [5-8] which occurred in our patient. This complication can be related to an inadequate surgical process or perivalvular fibrous tissue contracture. In a buphthalmic eye, there is relative elastic recoil that occurs when the intraocular pressure is normalized. Most probably, tube rotation occurs around the scleral spur, and the drainage tube ends up in a far more anterior position closer to the corneal endothelium surface. Placing these tubes close to the iris as possible may compensate for the expected anterior rotation after surgery in these eyes.

This case report confirmed that the Ahmed Glaucoma Valve may be safe and effective treatment for traumatic glaucoma in children, despite its postoperative complications. This procedure may be used to treat complicated glaucoma in eyes with previous failed filtering procedures.

\section{References}

1. Morad Y, Donaldson CE, Kim YM, Abdolell M, Levin AV (2003) The Ahmed drainage implant in the treatment of pediatric glaucoma. Am J Ophthalmol 135: 821-829.

2. Djodeyre MR, Peralta Calvo J, Abelairas Gomez J (2001) Clinical evaluation and risk factors of time to failure of Ahmed Glaucoma Valve implant in pediatric patients. Ophthalmology 108: 614-620.

3. Hamush NG, Coleman AL, Wilson MR (1999) Ahmed glaucoma valve implant for management of glaucoma in Sturge-Weber syndrome. Am J Ophthalmol 128: $758-760$.

4. Rosbach J, Choritz L, Pfeiffer N, Thieme H (2013) [Clinical results of encapsulated bleb removal after Ahmed glaucoma valve implants]. Ophthalmologe 110: $722-727$.

5. Wu SC, Huang SC, Lin KK (2003) Clinical experience with the Ahmed glaucoma valve implant in complicated glaucoma. Chang Gung Med J 26: 904-910.

6. Montañez FJ, Laso E, Suñer M, Amaya C (2005) [Ahmed drainage device implant. Our experience between 1995 and 2003]. Arch Soc Esp Oftalmol 80: $239-244$

7. Coleman AL, Smyth RJ, Wilson MR, Tam M (1997) Initial clinical experience with the Ahmed Glaucoma Valve implant in pediatric patients. Arch Ophthalmol 115: 186-191.

8. Englert JA, Freedman SF, Cox TA (1999) The Ahmed valve in refractory pediatric glaucoma. Am J Ophthalmol 127: 34-42.

9. Ayyala RS, Zurakowski D, Smith JA, Monshizadeh R, Netland PA, et al (1998) A clinical study of the Ahmed glaucoma valve implant in advanced glaucoma. Ophthalmology 105: 1968-1976.

10. Francis BA, Cortes A, Chen J, Alvarado JA (1998) Characteristics of glaucoma drainage implants during dynamic and steady-state flow conditions. Ophthalmology 105: 1708-1714 